\title{
Association of Anti-oxidative Capacity of HDL With Subclinical Atherosclerosis in Non-alcoholic Fatty Liver Disease
}

\section{Sara Karami}

Semnan University of Medical Sciences and Health Services Hossein Poustchi

Tehran University of Medical Sciences

Negar Sarmadi

Semnan University of Medical Sciences and Health Services

Amir Reza Radmard

Tehran University of Medical Sciences

\section{Fatemeh Ali Yari}

Semnan University of Medical Sciences and Health Services

Abbas Pakdel

Semnan University of Medical Sciences and Health Services

Parisa Shabani ( $\nabla$ parisa83j@gmail.com )

Semnan University of Medical Sciences and Health Services

\section{Research}

Keywords: HDL oxidative index, Non-alcoholic fatty liver disease, Cardiovascular disease, Carotid intimamedia thickness

Posted Date: August 17th, 2021

DOI: https://doi.org/10.21203/rs.3.rs-806043/v1

License: (c) (1) This work is licensed under a Creative Commons Attribution 4.0 International License. Read Full License 


\section{Abstract}

Background: Non-alcoholic fatty liver disease (NAFLD) patients are at the substantial risk for the for developing cardiovascular disease (CVD). High-density lipoprotein (HDL) is well known to have protective effects against the development of atherosclerotic CVD. One of the major antiatherogenic effect of HDL is its anti-oxidative function.

Objectives: This study investigated the association of anti-oxidative function of HDL with NAFLD and CVD risk.

Methods: A total of 143 subjects including 51 NAFLD and 92 control subjects were included in this crosssectional study. $\mathrm{HDL}$ oxidative index $(\mathrm{HOI})$ was determined spectrophotometrically using a cell-free method in the presence of a fluorescent substrate dichlorofluorescein diacetate (DCFDA). Paraoxonase 1 (PON1) activity, superoxide dismutase (SOD) activity and malondialdehyde (MDA) plasma levels were assessed in both groups.

Results: The subjects with impaired $\mathrm{HDL}$ anti-oxidative function ( $\mathrm{HOI} \geq 1)$ had lower PON1 paraoxonase activity, lower SOD activity, higher MDA levels and higher carotid intima-media thickness (cIMT) values compared to the controls. $\mathrm{HDL}$ oxidative index $(\mathrm{HOI})$ was positively correlated with MDA levels and cIMT and negatively correlated with SOD activity. NAFLD patients with $\mathrm{HOI} \geq 1$ had elevated serum levels of liver enzymes and higher LS values.

Conclusions: Alteration in circulating oxidative stress markers may diminish the anti-oxidative function of HDL. Impaired anti-oxidative capacity of HDL might be related to NAFLD severity and subclinical atherosclerosis in NAFLD patients.

\section{Introduction}

Non-Alcoholic Fatty Liver Disease (NAFLD) is the most leading cause of chronic liver disease which affects an estimated $20-30 \%$ of the general population (1). NAFLD is strongly associated with the other metabolic conditions such as obesity, type 2 diabetes mellitus, and cardiovascular diseases (1). Despite liver-related morbidity and mortality, cardiovascular disease (CVD) is the most important cause of mortality in the NAFLD population (2). Population based studies demonstrated increased CVD risk in NAFLD patients compared with matched healthy controls. It appears that NAFLD is mechanistically involved in the atherosclerosis development (3).

NAFLD patients exhibit an atherogenic dyslipidemia that is characterized by hypertriglyceridemia, low levels of high-density lipoprotein cholesterol (HDL-C), and high levels of low-density lipoprotein cholesterol (LDL-C) (4). Several studies demonstrated an association of higher plasma high-density lipoprotein (HDL) cholesterol levels with a lower risk of atherosclerotic cardiovascular disease (CVD) morbidity and mortality. However, there are multiple clinical observations which failed to support the causal relationship between $\operatorname{HDL-C}$ and $\operatorname{CVD}(5,6)$. This has led to the conception that the HDL particle 
functionality might be a better predictor of CDV risk than HDL cholesterol mass levels (7). HDL particle has numerous antiatherogenic functions. One of the important anti-atherogenic properties of HDL is its anti-oxidative function, the ability to suppress LDL oxidation which in turn decreases the atherogenicity of LDL particles (8).

Several studies addressed the anti-oxidative function of HDL in CVD and other disorders with high cardiovascular risk (8-11). The anti-oxidative property of HDL was not significantly different in non-MI patients and MI patient with or without ST elevation (8). The subjects with stable coronary artery disease had a similar HDL anti-oxidative function compared to the controls. However, the subjects who had acute coronary syndrome had less anti-oxidative capacity compared with the control subjects (9). Comparison of HDL anti-oxidative function in diabetic patients and healthy volunteers showed an impaired HDL antioxidative function in the diabetic patients (10). Additionally, the anti-oxidative function of HDL was impaired in the patients with PCOS (11). Previous studies showed impaired HDL cholesterol efflux capacity in NAFLD $(12,13)$. However, no study evaluated the anti-oxidative function of HDL in NAFLD patients.

Increasing data have demonstrated that NAFLD patients have abnormal circulating markers of oxidative stress, such as increased malondialdehyde (MDA), superoxide dismutase activity and PON1 activity (14, 15). Having a disturbed anti-oxidative system along with a high risk of CVD in these patients suggest that impaired antioxidant activities of $\mathrm{HDL}$, as an important player in CVD, may be the potential link between these phenomena. In this study, we aimed to investigate the relationship between HDL anti-oxidative capacity, NAFLD markers and carotid intima-media thickness (cIMT) in NAFLD.

\section{Material And Methods}

In the current study, 92 Controls and 51 NAFLD patients were selected from from Golestan Cohort Study (16). The was a cross-sectional study and approved by the medical ethics committee of the Semnan University of Medical Sciences (ethics code: IR.SEMUMS.REC.1397. 331) and all participants gave written informed consent. All participants were male, aged 50-81 years old. The diagnosis of NAFLD was based on abdominal ultrasonography. Subjects were excluded if they met one of the following criteria: A history of alcohol abuse $(30 \mathrm{~g} / \mathrm{d})$, diabetes, viral hepatitis, autoimmune liver disease, hemochromatosis, Wilson's disease. None of the patients were taking medication that has been reported to induce hepatitis.

Ultrasound assessment was performed using an Accuvix XQ ultrasound unit (Medison, South Korea) equipped with a 3-7 MHz curved-array and a 5-12 MHz linear-array transducer for the evaluation of liver, abdominal fat and carotid arteries as previously described (16). Visceral Adipose Tissue thickness (VAT) was measured between the anterior wall of the aorta and the internal face of the rectus abdominis muscle perpendicular to the aorta. (16). Carotid intima-media thickness (cIMT) was assessed as the distance between the lumen-intima interface and the media- adventitia interface, measured at its thickest point on the distal (far) wall of the common carotid arteries, $1.5-2 \mathrm{~cm}$ proximal to the carotid bulb. The average of right and left sides was used for cIMT analysis (16). Liver stiffness (LS) was 
measured by transient elastography using the FibroScan 502 machine (EchoSense, Paris, France, 5MHz) (16).

Anthropometric parameters were measured in accordance with the standardized protocols. The body mass index (BMI) was calculated as body weight in kilograms divided by the square of height in meters $(\mathrm{kg} / \mathrm{m} 2)$. WC was measured mid-way between the lowest rib and the iliac crest. Blood pressure was measured after 5 min rest at the left arm in a sitting position using an automatic manometer.

Fasting blood samples were obtained from the participants following an overnight fasting. Serum and plasma were isolated and frozen in aliquots at $-80^{\circ} \mathrm{C}$ for further analysis. Biochemical parameters including Fasting blood glucose (FBG), serum total cholesterol (TC), triglycerides (TG), high-density lipoprotein cholesterol (HDL-C), low-density lipoprotein cholesterol (LDL-C), and levels of alanine amino transferase (ALT), aspartate amino transferase (AST), gamma glutamyl transferase (GGT) were measured by automated enzymatic methods using commercial kits (Pars Azmoon, Iran).

Paraoxonase 1 (PON1) enzymatic activity was measured according to a method described before. Paraoxon (Sigma-Aldrich, Germany) was used as the substrate and PON1 activity was assessed by measuring the rate of substrate hydrolysis to $\mathrm{p}$-nitrophenol. Briefly, $5 \mu \mathrm{L}$ of plasma was added to a mixture of $1.0-\mathrm{mM}$ paraoxon in $195 \mu \mathrm{L}$ of $50 \mathrm{mM}$ glycine buffer containing $1 \mathrm{mM}$ calcium chloride $(\mathrm{pH}$ 10.5) and incubated at $37^{\circ}$ for 10 minutes in 96 well plates. The formation of p-nitrophenol was recorded at $412 \mathrm{~nm}$ and activity was expressed as $\mu \mathrm{mol}$ p-nitrophenol/L/plasma/min (17).

Superoxide dismutase (SOD) activity was measured by a SOD activity assay kit (Teb Pazhouhan Razi, Iran) following the manufacturer's instructions. The kit measures SOD activity by utilizing tetrazolium salt as a substrate which converts to a water-soluble formazan dye upon reduction by superoxide anion. The formazan formation rate is inhibited by the presence of SOD in the samples.

Plasma MDA levels were determined using an MDA assay kit (Teb Pazhouhan Razi, Iran) following the manufacturer's instructions. The method principle was based on the spectrophotometric measurement of the colored compound that is formed during the reaction of MDA and thio- barbituric acid (TBA) at 530 $\mathrm{nm}$.

The high-density oxidative index $(\mathrm{HOI})$ measures the ability of apoB-depleted plasma to inhibit $\mathrm{LDL}$ oxidation in the presence of dichlorofluorescein diacetate (DCFDA) (Sigma-Aldrich, Germany). A cell-free assay was performed as previously described with some modifications. Before analysis, apolipoprotein (apo) B depleted plasma was prepared as previously described. Briefly $40 \mu \mathrm{L} 20 \%$ polyethylene glycol (Merck, Germany) in Glycine was added to $200 \mu \mathrm{L}$ plasma, mixed and incubated on ice for 30minute. Then the mixture was centrifuged at $2200 \mathrm{~g}$ for 30 minutes, the HDL-containing supernatant was collected, kept on ice, and used for the further measurements. LDL was obtained from (Man lab Co., Iran) which was isolated from pooled fresh sera of healthy volunteers by sequential ultracentrifugation. LDL was diluted in PBS to a final cholesterol concentration of $100 \mu \mathrm{g} / \mathrm{mL}$ and oxidized by CuSO4 $(100 \mu \mathrm{mol} / \mathrm{L}$ ) for 6 hours at $37^{\circ} \mathrm{C}$. Oxidized LDL with a concentration of $1.4 \mu \mathrm{g} / \mathrm{ml}$, DCFDA with a concentration of 
$0.725 \mu \mathrm{g} / \mathrm{ml}$ and apo B-depleted plasma from the participants with PBS to a final volume of $175 \mu \mathrm{l}$ were added to the separate wells of a 96-well flat bottom polystyrene microtiter plates and incubated at $37^{\circ} \mathrm{C}$ for $60 \mathrm{~min}$. Fluorescence intensity was measured with a at an excitation wavelength of $485 \mathrm{~nm}$ and emission wavelength of $530 \mathrm{~nm}$ using the synergy h1 hybrid multi-mode microplate reader (BioTek, USA). The $\mathrm{HDL}$ oxidative index $(\mathrm{HOI})$ was calculated as the ratio of fluorescence intensity in the presence of apo-B-depleted plasma samples divided by the fluorescence intensity in the absence of apo-B-depleted plasma. An $\mathrm{HOI}<1$ was considered as anti-oxidative $\mathrm{HDL}$ function and an $\mathrm{HOI} \geq 1$ was considered as pro-oxidative HDL.

The Kolmogorov-Smirnov test was used to test for normal distribution of data. Categorical data are presented as percentages, continuous data as the mean \pm SD or median (IQR) as appropriate.

Comparison between two groups was performed using independent-sample $t$ test or Mann-Whitney $U$ test for continuous data and the chi-squared test for categorial data. Differences among the four subgroups as classified based on the presence of NAFLD and HOI were determined using the Kruskal-Wallis test. When analysis among the four groups was significant, pairwise tests were performed using the MannWhitney test. Pearson correlation was applied to determine the correlations between $\mathrm{HOI}$ and the other parameters in the whole study population. Significant differences were defined by $\mathrm{P}<0.05\left(^{*}\right)$ or $\left({ }^{*}\right), \mathrm{P}<$ $\left.0.01{ }^{(\star \star}\right)$ or $(\# \#)$, and $\mathrm{P}<0.001\left({ }^{(\star \star}\right)$ or $(\# \# \#)$.

All of the statistical analyses were performed using the IBM SPSS Statistic 27 and GraphPad prism 9.

\section{Results}

Basic clinical and laboratory characteristics of the study groups are presented in Table 1. BMI, waist circumference (WC), waist-to-hip ratio, fasting blood glucose (FBG), TG, visceral fat, AST, ALT, GGT, and LS were significantly higher in the NAFLD group compared with the control group. NAFLD patients had lower HDL-C than the control subjects. There was not any significant difference in age, total cholesterol (TC), LDL-C, and CIMT between the two groups. 
Table 1

Baseline characteristics

\begin{tabular}{|c|c|c|c|}
\hline Parameter & Control $(n=92)$ & NAFLD $(n=51)$ & P-value \\
\hline Age (years) & $59.0(55.0-66.0)$ & $56.0(54.0-61.0)$ & 0.273 \\
\hline BMI (kg/m2) & $24.83 \pm 3.62$ & $29.34 \pm 3.47$ & $<0.001$ \\
\hline $\mathrm{WC}(\mathrm{cm})$ & $93.29 \pm 11.12$ & $103.67 \pm 9.65$ & $<0.001$ \\
\hline WHR & $0.97(0.92-1.00)$ & $0.98(0.94-1.03)$ & 0.002 \\
\hline Weight (kg) & $68.75(61.00-79.50)$ & $80.75(73.50-89.00)$ & $<0.001$ \\
\hline $\mathrm{TC}(\mathrm{mg} / \mathrm{dl})$ & $205.51 \pm 39.40$ & $207.34 \pm 37.00$ & 0.779 \\
\hline LDL-C (mg/dL) & $121.61 \pm 33.76$ & $124.61 \pm 28.71$ & 0.594 \\
\hline $\mathrm{HDL}-\mathrm{C}(\mathrm{mg} / \mathrm{dL})$ & $57.49 \pm 12.58$ & $51.94 \pm 11.12$ & 0.007 \\
\hline $\mathrm{TG}(\mathrm{mg} / \mathrm{dL})$ & $112.0(82.0-158.0)$ & $148.5(112.5-186.0)$ & 0.016 \\
\hline Systolic blood pressure (mmHg) & $131.55 \pm 21.34$ & $144.96 \pm 23.74$ & 0.001 \\
\hline Diastolic blood pressure $(\mathrm{mmHg})$ & $79.96 \pm 11.03$ & $86.88 \pm 12.43$ & 0.001 \\
\hline $\mathrm{FBG}(\mathrm{mg} / \mathrm{dL})$ & $92.71 \pm 9.00$ & $99.57 \pm 11.21$ & $<0.001$ \\
\hline Visceral fat (\%) & $46.57 \pm 20.76$ & $70.26 \pm 18.01$ & $<0.001$ \\
\hline AST (U/L) & $18.5(15.0-22.0)$ & $22.0(19.5-27.0)$ & $<0.001$ \\
\hline ALT U/L) & $15.5(11.0-22.0)$ & $31.5(23.0-41.0)$ & $<0.001$ \\
\hline GGT (U/L) & $22.37(17.40-29.10)$ & $28.65(23.53-38.50)$ & $<0.001$ \\
\hline LS (kPa) & $3.80(3.30-4.30)$ & $5.05(4.25-6.60)$ & $<0.001$ \\
\hline clMT (mm) & $0.8(0.75-0.87)$ & $0.81(0.74-0.91)$ & 0.863 \\
\hline $\mathrm{HOI}$ & $0.92 \pm 0.19$ & $0.95 \pm 0.24$ & 0.405 \\
\hline $\mathrm{HOI} \geq 1$ frequency $(\%)$ & 32.96 & 40.38 & 0.468 \\
\hline SOD (U/mg protein) & $10.71 \pm 1.51$ & $8.60 \pm 2.44$ & $<0.001$ \\
\hline $\operatorname{MDA}(\mu \mathrm{M})$ & $33.29(25.08-42.33)$ & $34.02(30.40-47.74)$ & 0.771 \\
\hline \multicolumn{4}{|c|}{$\begin{array}{l}\text { Baseline characteristics according to disease state. Comparisons between groups were performed } \\
\text { using Independent Student's t-test or Mann-Whitney U test as appropriate. Continuous data are } \\
\text { expressed as mean } \pm \text { standard deviation (SD) or median and (interquartile range), categorical data as } \\
\text { percentages. BMI: Body mass index; WC: waist circumference; TC: Total cholesterol; LDL-C: Low } \\
\text { density lipoprotein cholesterol; HDL-C: High density lipoprotein cholesterol; TG: Triglycerides; AST; } \\
\text { aspartate amino transferase; ALT: alanine amino transferase; GGT: gamma glutamyl transferase; LS: } \\
\text { liver stiffness; cIMT: Carotid intima-media thickness; MDA: malondialdehyde; PON1: Paraoxonase 1; } \\
\text { SOD: superoxide dismutase; HOl: high-density oxidative index. } p<0.05 \text { was considered statistically } \\
\text { significant. }\end{array}$} \\
\hline
\end{tabular}




\begin{tabular}{|c|c|c|c|}
\hline Parameter & Control $(n=92)$ & NAFLD $(n=51)$ & P-value \\
\hline PON1 (U/L) & $24.44(17.77-31.11)$ & $42.21(27.77-52.22)$ & $<0.001$ \\
\hline Hypertension (\%) & 27.90 & 47.36 & 0.021 \\
\hline \multicolumn{4}{|c|}{$\begin{array}{l}\text { Baseline characteristics according to disease state. Comparisons between groups were performed } \\
\text { using Independent Student's t-test or Mann-Whitney U test as appropriate. Continuous data are } \\
\text { expressed as mean } \pm \text { standard deviation (SD) or median and (interquartile range), categorical data as } \\
\text { percentages. BMI: Body mass index; WC: waist circumference; TC: Total cholesterol; LDL-C: LoW } \\
\text { density lipoprotein cholesterol; HDL-C: High density lipoprotein cholesterol; TG: Triglycerides; AST; } \\
\text { aspartate amino transferase; ALT: alanine amino transferase; GGT: gamma glutamyl transferase; LS: } \\
\text { liver stiffness; cIMT: Carotid intima-media thickness; MDA: malondialdehyde; PON1: Paraoxonase 1; } \\
\text { SOD: superoxide dismutase; HOI: high-density oxidative index. } p<0.05 \text { was considered statistically } \\
\text { significant. }\end{array}$} \\
\hline
\end{tabular}

There was not any significant difference in the mean of HOI between NAFLD and control groups. We found that $61(66.30 \%)$ controls and $30(58.82 \%)$ patients had a preserved anti-oxidative function with an $\mathrm{HOI}<1$, and 31 (33.69\%) controls and $21(41.17 \%)$ patients presented pro-oxidative HDL serum measurements with an $\mathrm{HOI} \geq 1$. We stratified the subjects into two groups, $\mathrm{HOI}<1$ and $\mathrm{HOI} \geq 1$. The subjects with $\mathrm{HOI} \geq 1$ had higher levels of MDA $(P=0.043)$, lower PON1 activity $(P=0.028)$, lower SOD activity $(P=0.012)$, and higher clMT values $(P=0.022)($ Fig. 1$)$.

We further stratified the study population into 4 groups according to combined strata of status and $\mathrm{HOI}$ above and below 1. Interestingly, NAFLD patients with $\mathrm{HOI} \geq 1$ had higher $\mathrm{LS}(P=0.034)$, AST $(P=0.039)$, $\operatorname{ALT}(P=0.036)$ and cIMT $(P=0.042)$ levels than NAFLD patients with $\mathrm{HOI}<1$ (Fig. 2).

Correlation analysis demonstrated that $\mathrm{HDL}$ antioxidant capacity $(\mathrm{HOI})$ correlated positively with plasma MDA levels $(r=0.298, P=0.001)$ and $c I M T(r=0.197, P=0.025)$ in the whole population. There was a significant negative correlation between $\mathrm{HOI}$ and the SOD activity $(r=-0.242, \mathrm{P}=0.004)$ in the whole population (Table 2). 
Table 2

Correlation coefficients between $\mathrm{HOI}$ and metabolic and anthropometric

parameters

\begin{tabular}{|lll|}
\hline \multicolumn{3}{|c}{ parameters } \\
\hline Parameter & Correlation & P value \\
\hline PON1 & 0.298 & 0.001 \\
\hline SOD & -0.117 & 0.201 \\
\hline Age & -0.242 & 0.004 \\
\hline CIMT & 0.056 & 0.503 \\
\hline Visceral Fat & 0.188 & 0.031 \\
\hline WC & 0.011 & 0.909 \\
\hline BMI & 0.042 & 0.9 \\
\hline WHR & 0.012 & 0.892 \\
\hline AST & 0.059 & 0.488 \\
\hline ALT & 0.067 & 0.432 \\
\hline GGT & 0.075 & 0.374 \\
\hline LS & 0.062 & 0.474 \\
\hline FBG & 0.077 & 0.358 \\
\hline HDL-C & -0.044 & 0.603 \\
\hline LDL-C & 0.032 & 0.705 \\
\hline TG & -0.009 & 0.919 \\
\hline
\end{tabular}

\section{Discussion}

Cardiovascular diseases are the main cause of death in NAFLD patients (18). Impaired anti-oxidative capacity of HDL turned out to be a good predictor of cardiovascular disorders (19). Our findings showed that the subjects with an impaired anti-oxidative capacity of HDL tend to present higher oxidative stress markers. We also found that impaired anti-oxidative capacity of plasma HDL was associated with higher levels of hepatic fibrosis markers and higher cIMT in NAFLD.

A previous study on anti-oxidative properties of $\mathrm{HDL}$ in patients with coronary syndrome found higher $\mathrm{HOI}$ in patients with acute coronary syndrome or stable coronary artery disease compared with controls (9). Another study reported total HDL antioxidant capacity in systemic lupus erythematosus patients who have increased risk of cardiovascular diseases were significantly reduced compared to the controls (20). 
But there was no significant difference in anti-oxidative properties of HDL in MI patients with or without ST elevation and non-MI participants (8). We also did not find a significant difference in HOI values between the NAFLD and the control groups. The frequency of $\mathrm{HOI} \geq 1$ was higher in NAFLD compared to the controls, albeit not significant. Consistently, it has been reported that impaired anti-oxidative capacity of plasma HDL was more frequent in women with polycystic ovary syndrome (PCOS) compared with the control group (11). These conflicting results might be due to the different study populations and methods used in the studies.

In the current study, we found higher level of MDA in the NAFLD group compared to the control group. In parallel, previous studies reported increased levels of MDA in the NAFLD patients compared to controls $(14,15,21)$. Numerous pieces of evidence unraveled the important role of oxidative stress in the pathogenesis of NAFLD. Oxidative stress causes by the imbalance between the production of reactive oxygen species (ROS) and the intrinsic antioxidant capacity of a biological system (22). ROS oxidizes various important classes of biological molecules including lipids of all cellular membranes and lipoproteins (23). HDL oxidation generates hydroperoxides in HDL which subsequently produces several reactive intermediates such as MDA (23). A Previous study showed increased levels of MDA in HDL subfractions isolated from the plasma of acute coronary syndrome patients compared to control subjects and it was along with a pro-inflammatory effect of HDL (24). In consistent, we found higher levels of plasma MDA in the subjects with pro-oxidative $\mathrm{HDL}(\mathrm{HOI} \geq 1)$ compared to the subjects with $\mathrm{HOI}<1$. Moreover, our findings showed a significant positive correlation between $\mathrm{HOI}$ values and MDA levels.

Our results showed a higher level of PON1 paraoxonase activity in the NAFLD than the controls. Conversely, other studies showed lower PON1 serum concentration in the NAFLD group compared to the control group $(25,26)$. However, in consistent with our findings, a large population study which used fatty liver index for the diagnosis of NAFLD showed that in men PON-1 activity was significantly higher in the NAFLD population compared to the non-NAFLD population (27). PON1 has been shown to be critical for the anti-oxidative function of HDL particle. A previous study demonstrated a lower PON-1 activity in patients with an $\mathrm{HOI} \geq 1$ than the subjects with an $\mathrm{HOI}<1$ (7). Consistently, we found lower levels of PON1 paraoxonase activity in the $\mathrm{HO} \geq 1$ group compared to the $\mathrm{HOl}<1$ group.

With respect to SOD activity, we observed decreased activity of SOD in NAFLD patients. Similarly, other studies reported lower activity of SOD in NAFLD patients $(14,28,29)$. In our study, SOD activity was inversely correlated with fatty liver and obesity indices such as ALT, LS, BMI, WHR and FBS. A previous report of elevated levels of triglyceride in hepatic cells of SOD knockout mice suggests the contribution of SOD in the development of fatty liver (30). Our findings showed a lower SOD activity in the subjects with $\mathrm{HOI} \geq 1$ compared to the subjects with $\mathrm{HOI}<1$.

Collectively, higher MDA level, lower PON1 and lower SOD which reflects an impaired intrinsic antioxidant system in the subjects with $\mathrm{HOI} \geq 1$ confirms previous observations showing the relationship between oxidative stress and impaired HDL antioxidant capacity. As it was mentioned earlier, the oxidation of lipid 
and protein constituents in HDL is the pivotal cause of impaired HDL functions (31). On the flip side, it has been shown that HDL oxidation causes degradation of PON1 paraoxonase activity (32).

Moreover, we found that NAFLD patients with the impaired antioxidant function of $\mathrm{HDL}(\mathrm{HOI} \geq 1)$ had higher ALT, AST and LS. Previous studies in NAFLD patients demonstrated the association of circulating oxidative stress biomarkers with disease severity (22). In the light of the important role of oxidative stress in the progression of NAFLD, one can speculate that NAFLD patients with higher liver enzymes and liver fibrosis have higher levels of pro-oxidative and other detrimental factors which cause HDL dysfunctional. However, owing to the multiple anti-oxidative and anti-inflammatory properties of $H D L$, the possibility that an impaired anti-oxidative capacity of HDL may affect disease severity in NAFLD patients cannot be ruled out.

Finally, we observed higher levels of cIMT in the subjects with $\mathrm{HDL} \geq 1$ compared to the subjects with $\mathrm{HOI}<1$. We also found a significant positive correlation between $\mathrm{HOI}$ and cIMT. However, a previous study on young adults did not find an association between the impaired anti-oxidative capacity of HDL and CIMT (17). cIMT is considered as a surrogate marker of cardiovascular risk (33). Our findings regarding the association of $\mathrm{HOI}$ with cIMT supports the notion that impaired anti-oxidative capacity of HDL can be a good predictor of cardiovascular issues. Of note, we found that NAFLD patients with the impaired antioxidant function of $\mathrm{HDL}(\mathrm{HOI} \geq 1)$ had higher cIMT. This would suggest that the antioxidant function of HDL might be one of the potential mediators of the relationship between NAFLD and CVD.

There are some limitations to this study. The study was cross sectional in nature, which does not provide information about possible causal relationships among NAFLD markers, HDL anti-oxidative capacity and oxidative stress markers. So, further prospective studies are required to specifically address the potential role of HDL anti-oxidative function in the development and progression of NAFLD. We used apolipoprotein B-depleted plasma samples instead of isolated HDL to measure the anti-oxidative capacity of HDL. Polyethylene glycol precipitation is considered as a reproducible and rapid method to extract HDL from plasma samples $(9,19)$, but there is a possibility that anti-oxidative effects are influenced by other proteins rather than HDL associated proteins. However, a previous study showed comparable $\mathrm{HOI}$ values determined with apo B depleted sample and ultracentrifugation isolated $\mathrm{HDL}$.

\section{Conclusions}

In summary, our results showed that impaired anti-oxidative capacity of HDL was associated with higher circulating levels of oxidative stress markers and cIMT, suggesting the possible contribution of disturbed anti-oxidative system in causing HDL dysfunctional which in turn can elevate the risk of CVD. The relationship between impaired anti-oxidative capacity of HDL and NAFLD markers suggest either this disease state might attenuate the anti-oxidative effect of $\mathrm{HDL}$ or $\mathrm{HDL}$ anti-oxidative functionality might contribute to NAFLD pathogenesis. In perspective, further studies are required to elucidate the causal relationship among oxidative stress, the anti-oxidative capacity of HDL, NAFLD pathogenesis and risk of CVD in NAFLD. 


\section{Abbreviations}

NAFLD: Non-alcoholic fatty liver disease

CVD: Cardiovascular disease

HDL: High-density lipoprotein

DCFDA: Dichlorofluorescein diacetate

PON1: Paraoxonase 1

HOI: HDL oxidative index

SOD: Superoxide dismutase (SOD)

MDA: Malondialdehyde (MDA)

CIMT: Carotid intima-media thickness

LDL-C: low-density lipoprotein cholesterol

VAT: Visceral Adipose Tissue thickness

LS: Liver stiffness

FBG: Fasting blood glucose

TC: Total cholesterol

TG: Triglyceride

HDL-C: high-density lipoprotein cholesterol

ALT: Alanine amino transferase

AST: Aspartate amino transferase

GGT: Gamma glutamyl transferase

\section{Declarations}

\section{Ethics approval and consent to participate}

All participants gave written informed consent. This study was approved by the medical ethics committee of the Semnan University of Medical Sciences (ethics code: IR.SEMUMS.REC.1397. 331). 


\section{Consent for publication}

Not applicable

\section{Availability of data and materials}

The datasets used and/or analyzed during the current study are available from the corresponding author on reasonable request.

\section{Competing interests}

The authors declare that they have no competing interests.

\section{Funding}

This work was supported by the Semnan University of Medical Sciences. The funding body played no role in the design of the study and collection, analysis, and interpretation of data and in writing the manuscript.

\section{Authors' contributions}

PS designed the study, analyzed the data and wrote the manuscript. AP and HP supervised the study and edited the manuscript. ARR did ultrasonography and fibroscan. SK, FAY, and NS performed the experiments and collected the human subjects. All authors approved the manuscript.

\section{Acknowledgments}

The research presented in this article is part of the dissertation of Sara Karami to receive a master's degree in clinical biochemistry. The authors wish to thank the study participants, doctors, and coresearchers of Digestive Diseases Research Institute of Tehran University of Medical Sciences.

\section{References}

1. Vernon G, Baranova A, Younossi Z. Systematic review: the epidemiology and natural history of nonalcoholic fatty liver disease and non-alcoholic steatohepatitis in adults. Alimentary pharmacology \& therapeutics. 2011;34(3):274-85.

2. McCullough A, Previs SF, Dasarathy J, Lee K, Osme A, Kim C, et al. HDL flux is higher in patients with nonalcoholic fatty liver disease. American Journal of Physiology-Endocrinology and Metabolism. 2019;317(5):E852-E62.

3. Gao S, Ramen K, Yu S, Luo J. Higher non-HDL-cholesterol to HDL-cholesterol ratio is linked to increase in non-alcoholic fatty liver disease: secondary analysis based on a longitudinal study. International Journal of Clinical and Experimental Pathology. 2020;13(10):2569. 
4. Wang K, Shan S, Zheng H, Zhao X, Chen C, Liu C. Non-HDL-cholesterol to HDL-cholesterol ratio is a better predictor of new-onset non-alcoholic fatty liver disease than non-HDL-cholesterol: a cohort study. Lipids in health and disease. 2018;17(1):1-8.

5. Rader DJ. New therapeutic approaches to the treatment of dyslipidemia. Cell metabolism. 2016;23(3):405-12.

6. Triolo M, Annema W, Dullaart RP, Tietge UJ. Assessing the functional properties of high-density lipoproteins: an emerging concept in cardiovascular research. Biomarkers in medicine. 2013;7(3):457-72.

7. Schrutka L, Distelmaier K, Hohensinner P, Sulzgruber P, Lang IM, Maurer G, et al. Impaired HighDensity Lipoprotein Anti-Oxidative Function Is Associated With Outcome in Patients With Chronic Heart Failure. J Am Heart Assoc. 2016;5(12).

8. Annema W, Willemsen HM, de Boer JF, Dikkers A, van der Giet M, Nieuwland W, et al. HDL function is impaired in acute myocardial infarction independent of plasma HDL cholesterol levels. Journal of clinical lipidology. 2016;10(6):1318-28.

9. Patel PJ, Khera AV, Jafri K, Wilensky RL, Rader DJ. The anti-oxidative capacity of high-density lipoprotein is reduced in acute coronary syndrome but not in stable coronary artery disease. Journal of the American College of Cardiology. 2011;58(20):2068-75.

10. Morgantini C, Natali A, Boldrini B, Imaizumi S, Navab M, Fogelman AM, et al. Anti-inflammatory and antioxidant properties of HDLs are impaired in type 2 diabetes. Diabetes. 2011;60(10):2617-23.

11. Zhang J, Zhang Y, Liu H, Bai H, Wang Y, Jiang C, et al. Antioxidant properties of high-density lipoproteins are impaired in women with polycystic ovary syndrome. Fertility and sterility. 2015;103(5):1346-54.

12. Fadaei R, Poustchi H, Meshkani R, Moradi N, Golmohammadi T, Merat S. Impaired HDL cholesterol efflux capacity in patients with non-alcoholic fatty liver disease is associated with subclinical atherosclerosis. Sci Rep. 2018;8(1):11691.

13. van den Berg EH, Gruppen EG, Ebtehaj S, Bakker SJL, Tietge UJF, Dullaart RPF. Cholesterol efflux capacity is impaired in subjects with an elevated Fatty Liver Index, a proxy of non-alcoholic fatty liver disease. Atherosclerosis. 2018;277:21-7.

14. Arya A, Azarmehr N, Mansourian M, Doustimotlagh AH. Inactivation of the superoxide dismutase by malondialdehyde in the nonalcoholic fatty liver disease: a combined molecular docking approach to clinical studies. Archives of physiology and biochemistry. 2019:1-8.

15. Świderska M, Maciejczyk M, Zalewska A, Pogorzelska J, Flisiak R, Chabowski A. Oxidative stress biomarkers in the serum and plasma of patients with non-alcoholic fatty liver disease (NAFLD). Can plasma AGE be a marker of NAFLD? Oxidative stress biomarkers in NAFLD patients. Free radical research. 2019;53(8):841-50.

16. Merat S, Poustchi H, Hemming K, Jafari E, Radmard AR, Nateghi A, et al. PolyPill for Prevention of Cardiovascular Disease in an Urban Iranian Population with Special Focus on Nonalcoholic 
Steatohepatitis: A Pragmatic Randomized Controlled Trial within a Cohort (Polylran - Liver) - Study Protocol. Arch Iran Med. 2015;18(8):515-23.

17. Breton CV, Yin F, Wang X, Avol E, Gilliland FD, Araujo JA. HDL anti-oxidant function associates with LDL level in young adults. Atherosclerosis. 2014;232(1):165-70.

18. Lee SB, Park G-M, Lee J-Y, Lee BU, Park JH, Kim BG, et al. Association between non-alcoholic fatty liver disease and subclinical coronary atherosclerosis: an observational cohort study. Journal of hepatology. 2018;68(5):1018-24.

19. Schrutka L, Distelmaier K, Hohensinner P, Sulzgruber P, Lang IM, Maurer G, et al. Impaired highdensity lipoprotein anti-oxidative function is associated with outcome in patients with chronic heart failure. Journal of the American Heart Association. 2016;5(12):e004169.

20. Gaál K, Tarr T, Lőrincz H, Borbás V, Seres I, Harangi M, et al. High-density lipopoprotein antioxidant capacity, subpopulation distribution and paraoxonase-1 activity in patients with systemic lupus erythematosus. Lipids in health and disease. 2016;15(1):1-8.

21. Ito F, Ito T. High-density lipoprotein ( $\mathrm{Hdl}$ ) triglyceride and oxidized HDL: new lipid biomarkers of lipoprotein-related atherosclerotic cardiovascular disease. Antioxidants. 2020;9(5):362.

22. Chen Z, Tian R, She Z, Cai J, Li H. Role of oxidative stress in the pathogenesis of nonalcoholic fatty liver disease. Free Radical Biology and Medicine. 2020;152:116-41.

23. Ito F, Ito T, Suzuki C, Yahata T, Ikeda K, Hamaoka K. The application of a modified d-ROMs test for measurement of oxidative stress and oxidized high-density lipoprotein. International journal of molecular sciences. 2017;18(2):454.

24. Carnuta MG, Stancu CS, Toma L, Sanda GM, Niculescu LS, Deleanu M, et al. Dysfunctional highdensity lipoproteins have distinct composition, diminished anti-inflammatory potential and discriminate acute coronary syndrome from stable coronary artery disease patients. Scientific reports. 2017;7(1):1-13.

25. Milaciu MV, Vesa ȘC, Bocșan IC, Ciumărnean L, Sâmpelean D, Negrean V, et al. Paraoxonase-1 serum concentration and PON1 gene polymorphisms: relationship with non-alcoholic fatty liver disease. Journal of clinical medicine. 2019;8(12):2200.

26. Samy W, Hassanian MA. Paraoxonase-1 activity, malondialdehyde and glutathione peroxidase in non-alcoholic fatty liver disease and the effect of atorvastatin. Arab journal of gastroenterology. 2011;12(2):80-5.

27. van den Berg EH, Gruppen EG, James RW, Bakker SJL, Dullaart RPF. Serum paraoxonase 1 activity is paradoxically maintained in nonalcoholic fatty liver disease despite low HDL cholesterol. J Lipid Res. 2019;60(1):168-75.

28. Koruk M, Taysi S, Savas MC, Yilmaz O, Akcay F, Karakok M. Oxidative stress and enzymatic antioxidant status in patients with nonalcoholic steatohepatitis. Ann Clin Lab Sci. 2004;34(1):57-62.

29. Videla LA, Rodrigo R, Orellana M, Fernandez V, Tapia G, Quiñones L, et al. Oxidative stress-related parameters in the liver of non-alcoholic fatty liver disease patients. Clin Sci (Lond). 2004;106(3):2618. 
30. Chakraborty D, Lei XG. Development of Nonalcoholic Fatty Liver in Superoxide Dismutase-1 Knockout Mice Was Associated with Altered Expression of Hepatic Fibroblast Growth Factor-21. The FASEB Journal. 2016;30(S1):267.7-.7.

31. Monserrat-Mesquida M, Quetglas-Llabrés $M$, Abbate M, Montemayor S, Mascaró CM, Casares M, et al. Oxidative Stress and Pro-Inflammatory Status in Patients with Non-Alcoholic Fatty Liver Disease. Antioxidants (Basel). 2020;9(8).

32. Jaouad L, Milochevitch C, Khalil A. PON1 paraoxonase activity is reduced during HDL oxidation and is an indicator of HDL antioxidant capacity. Free Radic Res. 2003;37(1):77-83.

33. Willeit P, Tschiderer L, Allara E, Reuber K, Seekircher L, Gao L, et al. Carotid Intima-Media Thickness Progression as Surrogate Marker for Cardiovascular Risk: Meta-Analysis of 119 Clinical Trials Involving 100667 Patients. Circulation. 2020;142(7):621-42.

\section{Figures}



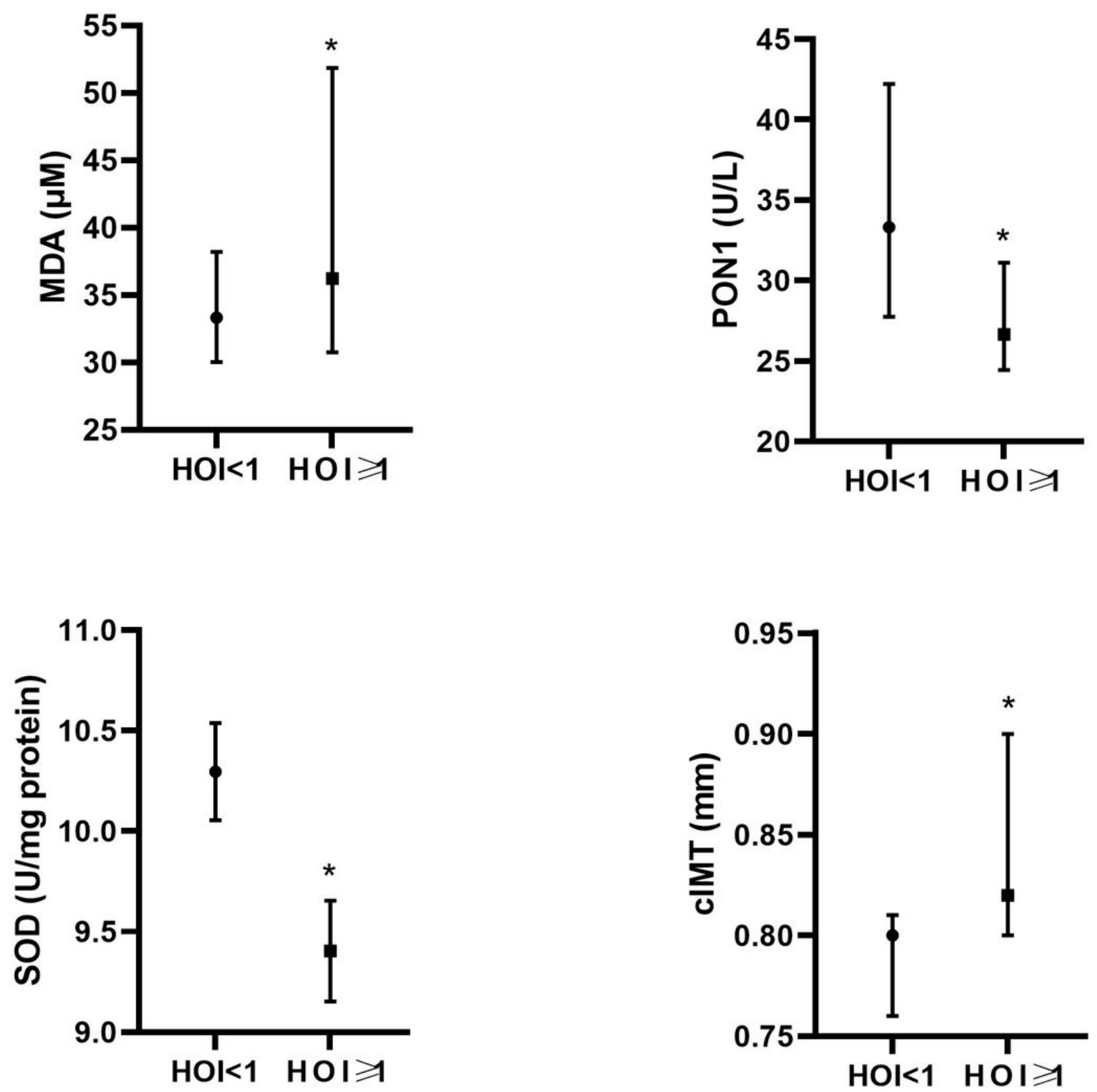

Figure 1

Comparison of CIMT, plasma levels of MDA, PON1 and SOD in the subjects with preserved anti-oxidative $\mathrm{HDL}$ capacity $(\mathrm{HOl}<1)$ and impaired anti-oxidative $\mathrm{HDL}$ capacity $(\mathrm{HOI} \geq 1)$. The differences between two groups were analyzed by independent Student's t-test or Mann-Whitney U test and were presented as mean \pm standard deviation or median with $95 \%$ confidence interval respectively. MDA: malondialdehyde, PON1: Paraoxonase 1, SOD: superoxide dismutase. $p<0.05$ was considered statistically significant. 

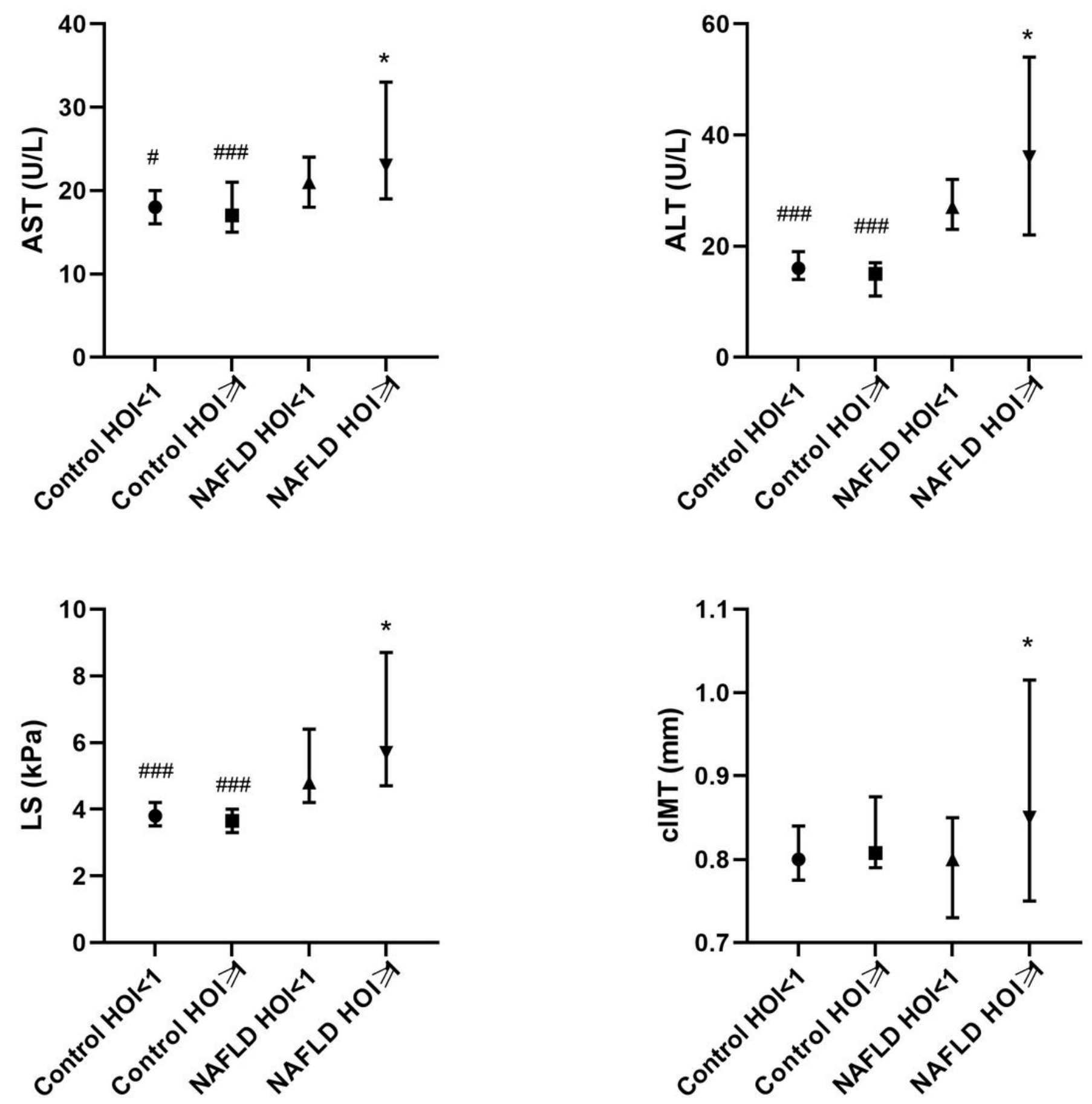

Figure 2

Comparison of AST, ALT, LS and CIMT in the subjects stratified by a HOI below or above 1 and disease state. Kruskal-Wallis test was used to compare among four groups and pairwise tests were performed using the Mann-Whitney test. Data were presented as median with $95 \%$ confidence interval. AST; aspartate amino transferase; ALT: alanine amino transferase; LS: liver stiffness, CIMT: Carotid intimamedia thickness. $\mathrm{p}<0.05$ was considered statistically significant. * indicates significant difference $\mathrm{HOI}<1$ and $\mathrm{HOI} \geq 1$. \# indicates significant difference between control and NAFLD groups. 